Sybille Isphording / Anna Buchheim /

Svenja Taubner

\title{
CHANGES IN REFLECTIVE \\ FUNCTIONING DURING PSYCHOANALYTIC PSYCHOTHERAPIES
}

This study examines how reflective functioning (RF) can be assessed in analytic sessions and throughout psychoanalytic psychotherapy. The goals are to replicate in part a study by Josephs and colleagues (2004) by applying the RF Scale to analytic sessions and to study fluctuations of RF within each session. Additionally, RF based on sessions was compared with the RF ratings based on the Adult Attachment Interview (AAl) during the course of two psychoanalytic psychotherapies with a duration of 240 hours. RF changes based on 10 sessions per patient, assessed at baseline and after 80,160 , and 240 hours of therapy, and RF changes based on AAl ratings measured at baseline and after 240 hours of therapy, and in one case at follow-up, were related to changes of symptoms and attachment classifications over time. Results showed that in both cases RF fluctuated within sessions. The average RF rating per session

Susanne Hörz-Sagstetter, Member, German Society for Psychoanalysis, Psychotherapy, Psychosomatics, and Depth Psychology (DGPT); Professor of Psychotherapy, Psychologische Hochschule, Berlin; Research Associate, Department of Psychology, Clinical Psychology, Psychoanalysis, and Psychodynamic Research section, Ludwig-Maximilians-Universität, Munich. Wolfgang Mertens, Professor Emeritus, Department of Psychology, Clinical Psychology, Psychoanalysis and Psychodynamic Research section, Ludwig-Maximilians-Universität, Munich; Training and Supervising Analyst (DGPT). Sybille Isphording, psychoanalytic candidate; private practice, Munich. Anna Buchheim, Member, IPA and DGPT; Professor and Chair for Clinical Psychology, Department of Psychology, Leopold-Franzens-Universität, Innsbruck. Svenja Taubner, Member, German Psychoanalytic Society; Chair for Clinical Psychology and Psychotherapy, Alpen-Adria-Universität, Klagenfurt, Austria.

This work was supported by the Köhler-Stiftung / Stifterverband für die Deutsche Wissenschaft, Baumgart-Stiftung, and the Bayerische Gleichstellungsförderung (BGF). The authors thank Susanne Stegmaier for contributing to the data analysis. Parts of this article were presented in a poster presentation at the Winter Meeting of the American Psychoanalytic Association, New York, January 13-17. Submitted for publication March 11, 2014. 
increased over the course of treatment, while the AAl-based RF rating needed longer to increase. Rather good correspondence was found between session-based RF ratings and independent AAl-based RF ratings. In both cases, changes in RF over time were compared to changes in attachment classification based on the AAl and to symptomatic change. Better correspondence between symptomatic and attachment changes was found with the AAl-based RF rating. It was tentatively interpreted that session-based RF ratings may represent a state of RF that is strongly influenced by the therapist-patient interaction, whereas AAIbased RF can be considered to have more trait characteristics.

Keywords: attachment, reflective functioning, psychoanalytic process research

$\mathbf{U}$ nderstanding the mind of self and others, or mentalizing, is considered a crucial element and mechanism of change in psychodynamic psychotherapies. The concept of mentalization, introduced by Fonagy and colleagues (2002), integrates cognitive theory-of-mind with attachment theory and psychoanalysis. As a bridging theory, mentalization integrates many facets of social cognition-including empathy, mindfulness, and psychological mindedness (Allen 2006). Choi-Kain and Gunderson (2008) have stressed that mentalization describes social cognition within several dimensions: (a) implicit and explicit aspects, (b) affective and cognitive contents, and (c) understanding self and other. Fonagy and colleagues (1998) developed a scale to assess reflective functioning (RF), referring to the psychological processes underlying the capacity to mentalize explicitly in the context of attachment relationships. The RF Scale, operationalized on a level from -1 (negative RF) to 9 (exceptional RF), is applied to the Adult Attachment Interview (AAI; George, Kaplan, and Main 1985) and has been used as an outcome instrument in psychotherapy research (Levy et al. 2006); however, it has rarely been applied to data sources other than the AAI (for an exception, see Josephs et al. 2004).

A growing body of research suggests that increase of mindfulness reduces stress, improves quality of life (Shapiro et al. 2005), and may prevent relapses in patients with depression (Segal, Williams, and Teasdale 2002). It has recently been suggested that improved RF might, like mindfulness, be an important component of successful psychotherapy outcome, 
especially with respect to achieving structural change in personality functioning. In general, improved mentalization is regarded as a common factor in therapeutic change independent of therapeutic school (Fonagy and Bateman 2006). In some patients structural change in terms of an increase in mentalization may be more important (e.g., in borderline personality disorder), whereas with other patients (e.g., those with depression or panic disorder) it could be more important to work with the content of representations (Fonagy et al. 1993). Similar to the uncertainty regarding the role of mentalization in therapeutic process, $\mathrm{RF}$ in psychotherapy research was assessed as an outcome variable or moderator, as well as a mediator, of psychotherapeutic change (Katznelson 2014). Effectiveness and efficacy studies have assessed changes in RF as an outcome variable in personality disorders (Levy et al. 2006; Vermote et al. 2010; Fischer-Kern et al. 2015), panic disorder (Rudden et al. 2006), depression (Karlsson and Kermott 2006; Taubner et al. 2015), and substance abuse (Grenyer and MiddlebyClements 2003), as well as changes of RF in mothers in parent-child therapy (Müller-Göttken, 2014). Three studies have assessed long-term changes in RF in single-case studies (Szecsödy 2008; Gullestad and Wilberg 2011; Josephs et al. 2004). Most studies have employed the gold standard by using the Adult Attachment Interview (AAI) to assess RF (Levy et al. 2006; Fischer-Kern et al. 2015; Taubner et al. 2015) or a shortened version of it (Rudden et al. 2006). Some authors have coded RF from therapy transcripts (Josephs et al. 2004; Karlsson and Kermott 2006) or used other interviews, such as the Object Relations Inventory (ORI) (Vermote et al. 2010) or the Parent Development Interview (PDI; MüllerGöttken et al. 2014).

Results revealed that although mentalization is considered a common factor in all therapeutic approaches, RF was increased especially in psychodynamic approaches. A significant increase of RF was reported after one year of transference-focused psychotherapy (TFP) but not in supportive or dialectical behavioral therapy (DBT) (Levy et al. 2006) or in nonmanualized therapy with experienced therapists from the community (FischerKern et al. 2015). A twelve-month inpatient and day-clinic treatment of patients with personality disorders revealed no improvement in RF (Vermote et al. 2010). However, this study used the ORI to assess RF, which is not validated on the AAI. Improvement of RF has been shown in chronically depressed patients after twenty-four months of psychoanalytic psychotherapy (Taubner et al. 2015) but not in patients with depression or 
anxiety disorder after short-term psychotherapies (cognitive behavioral, psychodynamic, or interpersonal) (Karlsson and Kermott 2006). However, psychodynamic panic-focused psychotherapy led to a significant increase of symptom-related RF in panic-disordered patients after twenty-four sessions, as against a relaxation intervention (Rudden et al. 2006). A shortterm psychodynamic intervention for children with depression and anxiety disorders, with accompanying parent therapy, revealed no changes in RF in mothers after forty-one weeks of psychotherapy (Müller-Göttken et al. 2014). Single-case studies have documented changes in RF after five years of psychoanalysis (Szecsödy 2008; Gullestad and Wilberg 2011). Grenyer and Middleby-Clements (2003) assessed changes in RF after sixteen sessions of psychotherapy or self-help-groups with cannabis-dependent patients. They reported that only patients in the supportive-psychodynamic group improved in RF. In summary, the evidence so far suggests that RF can be improved in diverse patient groups after long-term psychodynamic therapy, whereas psychodynamic inpatient treatment and short-term therapies have yielded mixed results.

Besides being an outcome factor, RF has been used to predict therapeutic outcome as a mediator. Müller et al. (2006) investigated a sample of twenty-four female patients with eating and depressive disorders. RF predicted improvement in overall mental conditions through a three-month inpatient therapy measured by Derogatis's Symptom Check List (SCL-90; Franke 1995). Most interestingly, RF predicted therapy success independent of the structural dimensions covered by the OPD (Operationalized Psychodynamic Diagnosis) Task Force (2008), which emphasizes its clinical independence. Further, RF was predictive of symptomatic changes in twenty depressed patients measured by the General Severity Index (GSI) after eight months of psychoanalytic therapy (Taubner et al. 2011). After follow-up at thirty-six months, RF measured from baseline was a significant predictor for changes in depression (Taubner et al. 2015). In contrast, Gullestad et al. (2013) could not find a general predictor effect of RF for the treatment of patients with borderline or avoidant personality disorder who were randomized in individual outpatient treatment or combined individual and group treatment in inpatient and outpatient settings. However, in this study RF moderated treatment effects and revealed that patients with low RF before treatment benefited from individual outpatient treatment, whereas patients with higher RF improved in both settings in psychosocial functioning. Thus, level of mentalization seems to be important tor therapy indication and treatment planning. 
One study (Levy et al. 2006) revealed a relationship between changes in $\mathrm{RF}$ and changes in attachment representation from disorganized to organized attachment patterns. Since RF and attachment were assessed from the same interview, it remains unclear if RF changes lead to changes in attachment or vice versa. However, on the basis of results from studies on symptomatic changes and RF, it has been hypothesized that changes in RF indicate structural changes in personality functioning that are followed by later symptomatic changes (Fischer-Kern et al. 2015). Thus, mentalization as measured with the AAI may be considered a mediator of psychotherapeutic changes, at least in psychodynamic psychotherapies (Katznelson 2014). This could be related to the pronounced importance of gaining insight or the ability to be insightful as a curative factor in psychodynamic therapies (Johansson et al. 2010).

Studies on the relationship between process and outcome that used session transcripts to score RF have yielded rather contradicting results. One study compared RF scores in one session from the early part and one session in the later part of the treatment, one using cognitive behavioral therapy (CBT) and one interpersonal psychotherapy (IPT) (Karlsson 2005; Karlsson and Kermott 2006). The average treatment length was 16.2 sessions in a sample with only female patients with major depression in a randomized clinical trial. In a second study, the research group investigated a sample of thirty cases of brief psychodynamic psychotherapy (BPDT) with an average treatment length of 15.8 sessions in a naturalistic design with a mixed sample of patients with depression, dysthymia, and generalized anxiety disorder. RF was assessed in both samples during the first, the fifth, and the fourteenth session of each treatment. Results from the two studies suggested that patients' RF, as measured through the discourse from therapy sessions, is stable (in CBT and BPDT) or decreases (IPT) during the treatments. High $\mathrm{RF}$ in the first session was related to good outcome, whereas low RF in the first session correlated with poor outcome. Bernbach et al. (2000) investigated the development of RF in twenty therapeutic dyads during over thirty sessions of brief relational therapy. In contrast to Karlsson and Karlsson (2006), they found that RF decreased during the process in patients with good outcome and remained stable in patients with poor outcome.

This approach includes methodological limitations that might explain the somewhat confusing results: first, the RF Scale has been validated on the AAI and, second, it may be difficult to separate the therapist's and the patient's contributions when scoring RF from session transcripts. This 
aspect was taken into account by Josephs et al. (2004). Their single-case study of a schizoid patient (Ms. Q.) contributed to a more standardized way of assessing RF from session transcripts by scoring blocks of 150 words that were rated for RF with a good level of interrater reliability (intraclass correlation coefficient $=.75$ on data from Ms. Q.). It was found that the patient's RF significantly improved over the period of treatment assessed in the study. However, it remains unclear how session-based RF is related to AAI-standardized RF. The present study was in part a replication of the single-case study by Josephs et al. (2004), but also adds to that study: it is the first to compare the application of RF as an outcome measure based on the AAI to RF as a process measure based on session transcripts of two psychoanalytic cases.

\section{GOALS OF THE STUDY}

The goals of the present study were to replicate parts of the work by Josephs and colleagues by applying the RF Scale to analytic sessions and to examine (a) fluctuations of RF within each session, (b) changes in RF based on sessions of two psychoanalytic psychotherapies over time, (c) RF ratings based on analytic sessions as compared with the gold-standard RF ratings based on the AAI in terms of external validation of the session-based approach, and (d) changes of RF as they relate to changes of attachment classifications on the AAI and to symptomatic changes based on the SCL-90-R.

We expected fluctuating levels of RF within the sessions, but an overall increase of RF based both on sessions and on the AAI over time as a marker of good outcome. As the research literature remains unclear on whether RF can be considered a moderator or mediator, we wanted to conduct an exploratory study on two single cases to better understand this question. Moreover, we hypothesized a good convergence between RF scores based on the two rating systems. And finally, we expected changes in the same direction in RF, attachment classifications, and symptoms.

\section{METHOD}

\section{Patients and Study}

Two cases from a German study (the Munich Attachment and Effectiveness Project) examining process and outcome of long-term psychoanalytic psychotherapies were randomly selected. This was done by 
blind choice of two treatments from those cases for which complete session material had been compiled and transcribed at the start of the present substudy. The Munich project is a prospective naturalistic psychotherapy study conducted by the Department of Psychology, University of Munich, in cooperation with a psychoanalytic institute (the Akademie für Psychoanalyse und Psychotherapie München). The project examines the psychotherapeutic process and outcome of twenty outpatient psychoanalytic psychotherapies, comparing results from process research with outcome measures. Two cases from this project, each studied for at least 240 hours and, if possible, in a follow-up interview, were chosen and examined.

Ms. A. was located at good to moderate level of personality functioning (score of 1.5 on a scale ranging from 1 to 4 ) according to the OPD-2 (OPD Task Force 2008) and with avoidant personality disorder (DSM-IV 301.82). This psychoanalytic psychotherapy lasted for eleven years (600 hours), with a setting of three times a week on the couch, which was modified to a weekly session after 370 hours.

Ms. B. was also located at good to moderate level of personality functioning (1.5). She was diagnosed with social phobia (DSM-IV 300.23) and a depressive episode, unspecified (DSM-IV 396.30). Ms. B. spent three years in psychoanalytic psychotherapy, with a total of 330 hours, three times a week on the couch. The OPD ratings were given by two independent raters; diagnoses were assigned clinically by the therapists. The study was approved by the ethics commission of the LudwigMaximilians-Universität in Munich. In accordance with the study's naturalistic design, participants were recruited by outpatient therapists and given a complete description of research. Those who gave written informed consent were included in the study.

\section{Measures}

Reflective functioning was coded according to the RF Scale (Fonagy et al. 1998), from the Adult Attachment Interview (AAI; George, Kaplan, and Main 1985) and therapy session transcripts. The RF Scale assesses whether subjects understand attachment-related experiences in terms of mental states. Their statements are coded on an 11-point-scale from antireflective (-1) to exceptionally reflective (9). Qualitative markers of RF are acknowledgment of the opacity of mental states, separateness of minds, developmental aspects, and efforts to understand behavior in terms of mental states. The RF Scale has been validated on the coherence 
scale of the AAI and shows good interrater reliability after training (Fonagy et al. 1998). Psychometric analysis has confirmed its unidimensional structure (Taubner et al. 2013). The authors describe two main areas: negative-to-low vs. average-to-high RF, with the level of 4 as the midpoint between them.

Each patient was interviewed using the AAI before and after 240 sessions of psychoanalytic therapy. The AAI consists of twenty questions asked in a set order with standardized probes. Subjects are asked to describe their childhood relationship with their parents, choosing five adjectives to describe each relationship and supporting these descriptors with specific memories (George, Kaplan, and Main 1985). To elicit attachment-related information they are asked how their parents responded to them when they were in physical or emotional distress (e.g., when as children they were upset, injured, or sick). They are also asked about memories of separation and loss, experiences of rejection, and times when they might have felt threatened, including, but not limited to, instances involving physical or sexual abuse. The interview requires that participants reflect on their parents' styles of parenting and that they consider how childhood experiences with their parents may have influenced their personality development. The technique has been described as having the effect of surprising the unconscious into revelation (George, Kaplan, and Main 1985) and allowing numerous opportunities for the interviewee to elaborate upon, contradict, or fail to support previous statements. Attachment classifications were also derived from the AAI. The AAI scoring system is designed to quantify the individual's current state of mind with respect to childhood attachment relationships. Scoring of the transcripts leads to an attachment classification: secure/autonomous (F), dismissive (Ds), preoccupied (E), or unresolved (U). The disorganized $U$ category is assigned a secondary organized classification (e.g., F, Ds, or E). Previous research has shown remarkable stability and predictive validity of the AAI (for a review, see Hesse 2010).

The Symptom Checklist-90-R (SCL-90-R; Franke 1995) is a 90-item self-report instrument that evaluates a broad range of psychological problems and symptoms of psychopathology. The General Severity Index (GSI) combines symptom severity from all 90 items.

\section{Procedure of Session-Based RF}

In the overall study, a host of methods were applied to session transcripts at six different points in time, among them the Psychotherapy 
Process Q-set (Jones 1985). For the present study, RF based on sessions was rated as follows: for each patient, ten audiotaped sessions from four points in time during the treatment (three at baseline, two at 80 hours of therapy, two at 160, and three at 240) were transcribed and analyzed. Thus, a total of twenty sessions were studied in detail. Following Josephs et al. (2004), each session was divided into 150-word blocks (+/-20 words to maintain the context of each text passage), and each of these word blocks was rated using the RF Scale. The ratings, based on the German translation of the RF manual (Fonagy et al. 1998), aimed at a careful adaptation of the manual's rating procedure to the rating of the the patient's reflective functioning in the analytic sessions. Ratings pertained to the patient's mentalizing capacity in the session, in response to both the therapist's interventions and the patient's narratives. In addition, a total RF score per session was given clinically, and from these clinical scores a mean score for each point in time was computed (i.e., mean score of the clinical scores of the sessions at baseline, at 80 hours, at 160 hours, and at 240 hours). Two independent raters who had reached good interrater agreement (ICC .886, using 235 blocks of 150 words each) were blinded for patient and session, and each rated all twenty sessions. After scoring was completed, ratings were assigned to patient and point in time and the research questions were examined. Accordingly, the rating procedure was applied to all twenty sessions in detail. The method is described by Isphording (2009) and Stegmaier (2007). In addition, an independent certified RF rater $^{1}$ blinded for patient and session assessed RF based on the AAI, which was conducted at baseline, after 240 hours, and at follow-up one year after the end of therapy. The same procedure was applied for the AAI: an independent certified AAIrater $^{2}$ rated the AAI transcripts from baseline, after 240 hours and at follow-up, blinded for patient and session. Patients completed a number of questionnaires; only the results of the SCL-90-R were examined for the present study.

${ }^{1}$ The RF-Scale was coded by a rater who had completed two three-day training workshops conducted by Fulvia Ronchi and Mary Target, and had achieved reliability on a set of fifteen transcripts.

${ }^{2}$ The AAI was scored by a rater who had completed a two-week training workshop conducted by Mary Main and Eric Hesse, and had achieved reliability on an extensive set of transcripts. 


\section{Figure 1. Fluctuation of RF Scale applied to sessions, across} ten sessions each for Ms. A. (left) and Ms. B. (right)

fluctuation of RF over 10 sessions

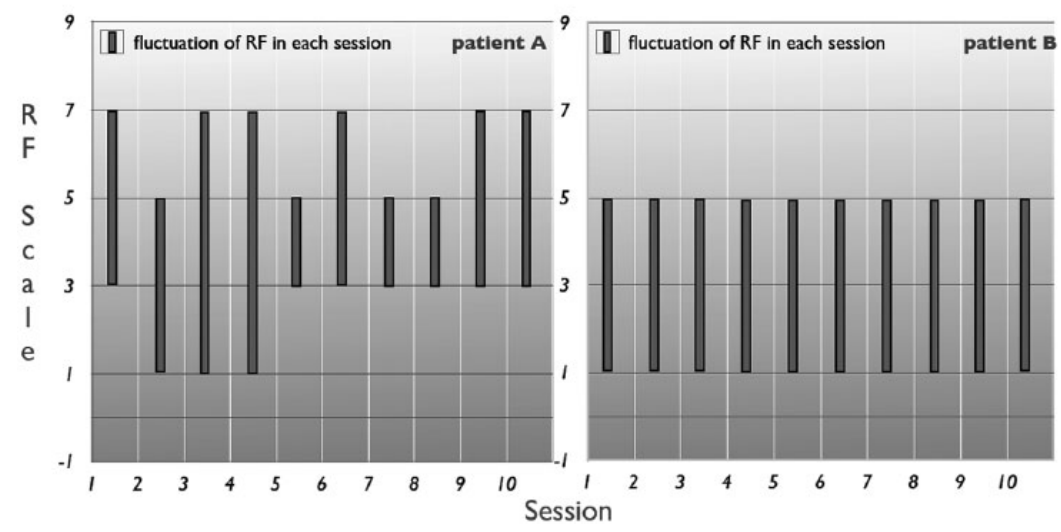

\section{RESULTS}

\section{Fluctuations of RF within Each Session}

For each patient, a session was chosen randomly from the twenty rated sessions to exemplify the procedure. The expected fluctuations of RF within each session were found in all of twenty sessions we examined. Figure 1 shows the fluctuations of session-based RF in the sessions. Fluctuations were studied as follows: each session was divided into 150word blocks and rated for RF, the RF scores were transferred to a table of $\mathrm{RF}$ scores per session, and then an overall RF score was given each session. Only after the blinded ratings were unmasked was the course of RF scores per session and across treatment analyzed. When analyzing the complete data set for the twenty sessions, it became clear that both patients showed a relative increase of moderate to high RF scores per session over the duration of the treatment (e.g., 16\% of RF ratings of 5 at a baseline session increased to $46 \%$ of such ratings at a session around 240 hours for Ms. B.). In Figure 1, fluctuations of RF per session and patient are presented in a vertical bar per session. For example, in Ms. A.'s first session, her level of RF fluctuated between 3 and 7. In the third session that was studied it fluctuated between 1 and 7 throughout the session.

One of the sessions shown in Figure 1 will serve as an example of how the RF score was applied on the patient's narrative throughout a session of 
Figure 2. RF Scale applied to 150 -word blocks in sessions, one session, Ms. A.

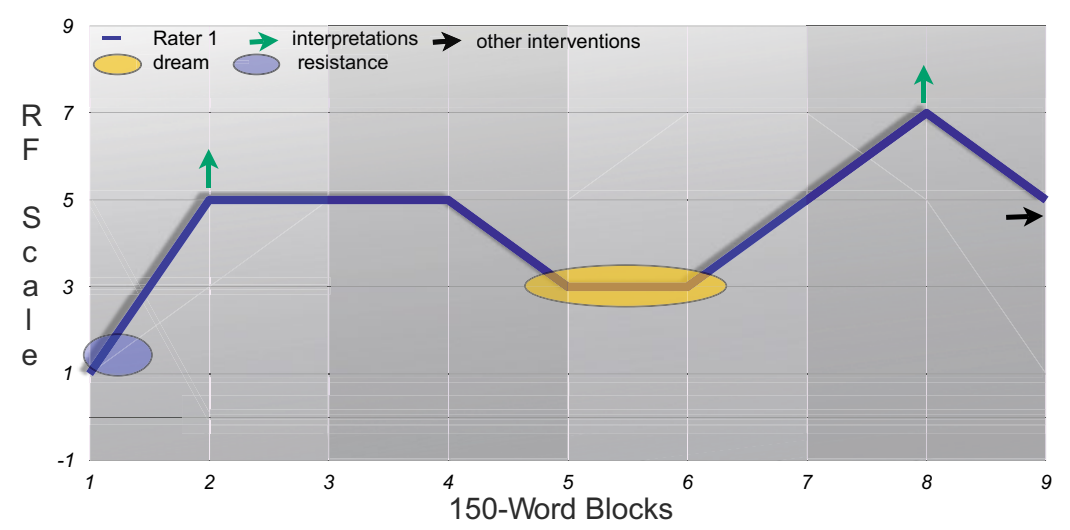

psychoanalytic psychotherapy. Session number 3 from Ms. A. (the third vertical bar in Figure 1) is analyzed in greater detail in Figure 2. The patient's statements are shown that were used to code RF; the therapist's interventions are not transcribed but summarized (see boxes below).

Ms. A. came into psychoanalytic psychotherapy reporting depressive symptoms, problems with sexuality, and difficulties distancing herself from others' wishes. In this session (the 88th) the therapist tries to talk about a massive resistance against the treatment, also shown in somatic symptoms, by making an interpretation at the beginning of the session and a dream interpretation at the end. The session ends with a clarification by the therapist coded as "other intervention." Figure 2 shows the course of interventions (gray arrows indicating interpretations, black arrows other interventions, e.g., clarifications) throughout the session, as well as changes in Ms. A.'s RF.

As the figure shows, resistance was coded for the patient at the beginning of the session: After coming late to session, she remains silent for seven minutes and also complains of feeling sick and having stomachaches for most of the session. Repeatedly, she says she has no idea where it's coming from, hence avoiding mentalization (word block [WB] 1; RF score of 1). The therapist interprets her resistance as stemming from anxiety over the prospect of becoming dependent on the therapeutic relationship, which leads to an increase of her RF level (WB 2; RF = 5): the patient is now able to reflect upon her resistance and withdrawal (WBs 2, 
$3,4)$. The following dream report relates to an abusive relationship in the past and leads to a decrease in RF (WBs 5, 6; RF =3). Reflecting on her own defensive processes was coded as an increase in the patient's RF (WB 7; RF $=5$ ) while she remained defensive against a deeper understanding of the dream. In his dream interpretation the therapist suggests to the patient that the present transference could be understood as transferring the abusive relationship from the past onto him. This interpretation has an effect on the patient and, now at the end of the session, she talks about her understanding of the meaning of her resistance and defensive processes. She realizes her fear of becoming dependent of the therapist and her fear of the abusive transference; she also links her fears to the somatic reactions at the beginning of the session (WB $8 ; \mathrm{RF}=7$ ). Finally, she states that she did not want to talk about this insight any further, hence defending against further elaboration (WB 9; $\mathrm{RF}=5$ ). The therapist ends the session with a clarification.

\section{Total RF Score for This Session}

The patient demonstrates a consistent way of mentalizing abilities that is accessible to the raters. In spite of her resistance at the start of the session, the total impression of the session corresponds to an ordinary reflective functioning (RF 5). Individual ratings mainly range around this ordinary level. Lower ratings relate to the dream report that reduces RF temporarily due to its descriptive nature. However, for the session as a whole, an RF rating of 5 was given.

The following excerpts from the session illustrate the rating process. Note that the number of words in each word block varies due to the translation from German into English. (Originally, each block contained 150 words.) For each block, the content is briefly summarized, followed by the RF rating and categories and excerpts from the patient's text in the session, omitting the therapist's statements (as was done by Josephs et al. 2004). The RF rating of each word block is shown in Figure 2.

Word block 1. The patient shows resistance against the therapy, avoids mentalization. Rating: lacking in $\mathrm{RF}=1 \mathrm{~A}$ (disavowal)

"I think I don't want to talk today. I came too late because I felt extremely sick earlier. . . . I don't know where that might have come from. I 
noticed, but . . . Sorry? . . . Yes, I don't even know, I think at quarter to eleven or something, when I came from the station, that's when it began .. . Hmm . . . (laughing:) I, I don't know. I can't tell. I find it pretty strange myself, but ... I don't know, what happened. If it might have to do with us here or not."

Word block 2. Therapist interprets the resistance as fear of dependency from the therapist. Interpretation raises RF. Rating: Ordinary $\mathrm{RF}=5 \mathrm{~A}$ (ordinary understanding; $\mathrm{RF}$ categories: recognition of the limitations on insight; awareness of the defensive nature of certain mental states)

“. . . also my stomach hurts, there is some pressure. . . it could be, well it is not conscious to me. It could be that it somehow is there, when I, ahem, when I notice I do not want to go into some topics at all, and, ahem, not at all, and . . yes, want to stay on the surface. There might be something like that. But completely clear-yes, that's it-no, I could not say it like that. . . Y Yes. I notice that I do avoid it completely."

Word block 3. Understanding that mental states affect behavior, thinking about own defenses. Rating: Ordinary RF $=5 \mathrm{~A}$ (ordinary understanding; RF categories: evaluating mental states from point of view of its impact on behavior of self and/or other; accurate attributions of mental states to others or self)

"it's all so difficult for me, even with topics that do not affect me at all. But I notice, I don't have any interest at all. It's been like that for a while, but somehow I noticed it especially during the weekend. That it happens not only here, but that generally, I am turning completely quiet. And I somehow have the feeling, everything is, I don't know, it's very difficult to describe, so meaningless. . . . And I also think, that I still have this idea, even if it's not true, but somehow I'm stuck with it, that, ahem, if I don't talk about it, it doesn't exist."

Word block 4. Defensive processes are described; understanding that content that was defended against finds expression in dreams. Rating: Ordinary $\mathrm{RF}=5 \mathrm{~A}$ (ordinary understanding; RF categories: awareness of the defensive nature of mental states) 
"So, I did not deal with it at all, because, ahem, then it is easier, or nothing happens or (mumbles) not wanting to think about yesterday evening. I even had a dream about it, but, ahem, I just want to let things happen. Not having to say anything, think anything and feel anything. . . . Ahem . . . I think I have repressed it again."

Word block 5. Dream report about someone stealing her wallet. Patient conveys an implicit knowledge of internal states. She takes on others' perspectives by projecting the guilt from the culprit to the victim (herself). Rating: Low $\mathrm{RF}=3 \mathrm{~A}$ (naive/simplistic; mental states are mentioned but not used for understanding)

". . . and suddenly I had the feeling, that something casually, somehow, that something casually had happened, and somehow I had a sense of happiness and looked to my purse and realized that the purse was open. . . it was clear to me that the other person thinks I might have stolen it. I knew it immediately and looked into the purse and it was true. The wallet was gone."

Word block 6. Dream report. Talks about volitions and feelings in the dream. Describes processes of internal states. Rating: Low RF $=3 \mathrm{~A}$ (naive/simplistic; several mental states are mentioned but not used for understanding)

"I did not do it then and the others laughed. That was very strange. I wanted to, and, and, something kept me from it after all. And, and then I, that somehow was very strange, in the moment that I somehow realized that my wallet was gone, and I wanted to get mad, and somehow felt panicked, and started thinking . . . in that moment somehow it flipped, and the danger and this, ahem, anger, was suddenly gone."

Word block 7. Patient understands her defensive processes regarding the end of the dream and explains these. Rating: Ordinary RF $=5 \mathrm{~A}$ (ordinary understanding; RF categories: awareness of the defensive nature of certain mental states)

"So for myself, I turned it into something completely positive, everything else would have been a total burden. It was unrealistic, but I somehow had no other, saw no other option. (Mumbles, breathes strongly) Well, what I noticed was that I simply felt that something was not okay." 
Word block 8. The therapist's interpretation leads to a high RF in the patient. She becomes aware of her resistance, her fear of dependence on the therapist. She understands that her feelings and thoughts have a defensive function and says that she hides her real mental states. This section shows exceptional insight into internal states that are difficult for the patient. Rating: High RF $=7$ (marked RF; RF categories: mental states as susceptible to disguise, awareness of the defensive nature of certain mental states)

"And then this ending: How I forcibly tried to find something unrealistic as ending. (Pause) But that was totally unrealistic for me. That was only in self-protection, but I felt that it was totally unrealistic. . . . I think I knew with your first question . . I knew with your first question, when I said I did not know, I really knew exactly what it was about, but I did not want to talk about it, or I did not want to address it and was hoping you would not either. Then I noticed it after all because, because I felt sick again."

Word block 9. The patient is aware of her defenses. She knows how to hide internal feelings and thoughts. Rating: Ordinary $\mathrm{RF}=5 \mathrm{~A}$ (ordinary understanding; RF categories: mental states as susceptible to disguise)

". . . when you spelled it out, with the open purse, and the robbery, what that could mean, that was my first association. But I thought I could sweep that, ahem, sweep that under the carpet."

Overall, the therapist's interventions had an influence on the patient's reflective functioning. The illustrating example from Ms. A.'s treatment (see Figure 2) shows a short-term increase of RF after a therapeutic intervention, as well as the interaction between her resistance and entanglement with the therapist and changes in RF.

Similarly, in the case of Ms. B., the patient's RF is influenced by the therapist's interventions. Figure 3 exemplifies the RF changes in Ms. B. within a session. In this session, the topics of conversation are various relationships. The patient begins by talking about a dream $(\mathrm{RF}=1-3)$ and increases her RF (from 3 to 5) following the therapist's questions about dream associations and developing her own ideas about the meaning of the symbols in her dream ("doll" = her wish for children). Following this 
Figure 3. RF Scale applied to 150-word blocks in sessions, one session, Ms. B.

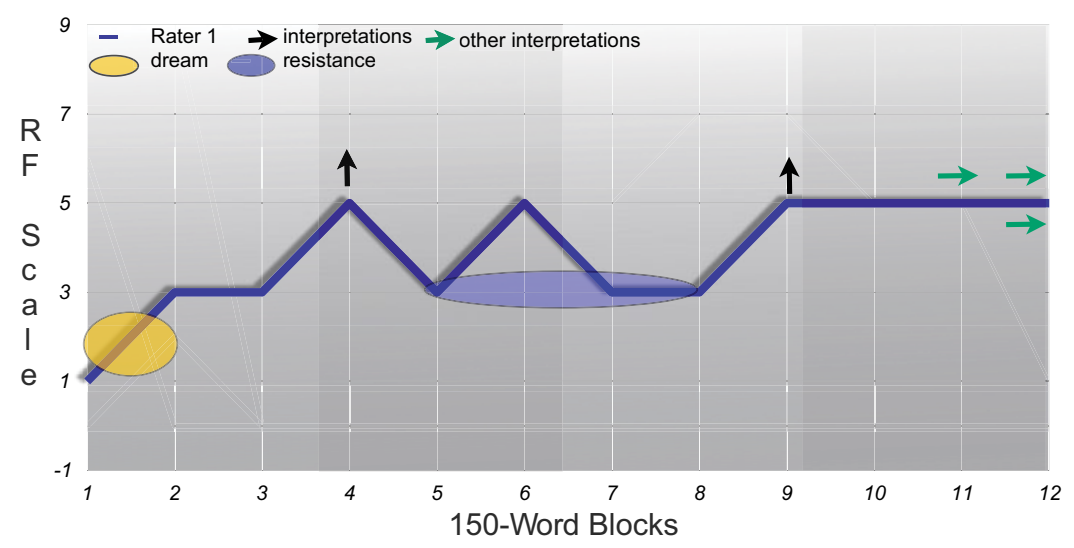

increase of RF, she talks about details of the previous day, seemingly as a resistance against deeper insight $(\mathrm{RF}=3)$. Again the therapist's questions lead to an increase of RF from 3 to 5 in the patient, by showing herself as thinking ("Hmm, I think I had not realized that in the moment, . . to be honest ... I was concentrating so much on my own thinking that it became clear to me only afterwards ... when we talked about it the next day"). The level of ordinary RF is maintained through the end of the session.

Hence, some associations between the therapist's interventions and fluctuating levels of RF in the patient were found in the two treatments we examined. In each case, ten sessions were studied in detail, and in every one of the examined sessions the patient's RF fluctuated and was influenced frequently by the therapist's interventions. For example, Ms. A. could temporarily increase her mentalization after the therapist's interpretations. As noted in the previous section, in Figure 2 it can be seen how the therapist's interpretation (the gray arrow) of the patient's fear of dependency can dissolve her resistance at the beginning of the session (the ellipse at the left) and increase her RF from 3 to 5 ("I wonder if your stomach pain might have something to do with the last session, in which you were conflicted about becoming more dependent of me or not. And also, if you could risk more dependency by committing more to our work or not"). Then during a dream report her RF declines to level 3, after which the dream interpretation at the end of the session leads to the patient's 
Figure 4. Mean scores of ratings of the RF Scale applied to sessions compared to the RF Scale applied to the AAI, changes in attachment representations and in SCL-90-R, Ms. A.

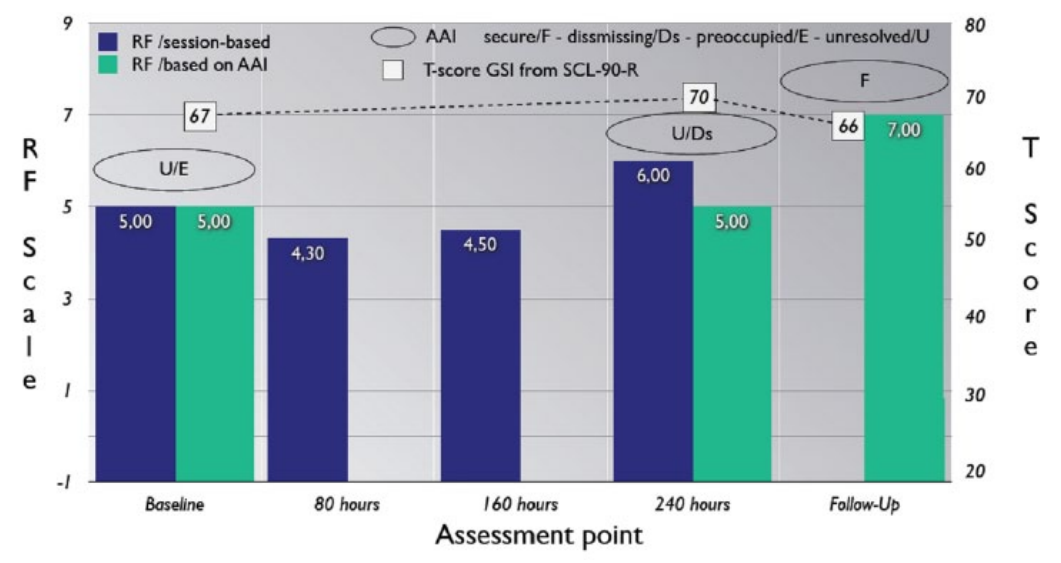

increased awareness of her resistance and an RF rating of 7. This example illustrates how the therapist's intervention can influence the patient's RF, in this case perhaps triggering higher RF by an interpretation. Of course, this finding cannot be generalized to other sessions or patients.

\section{Changes in Session-Based RF in Two Psychoanalytic Psychotherapies}

In the two cases, the average RF rating per session varied throughout the 240 hours of psychoanalytic psychotherapy. For Ms. A., the average rating of session-based RF scores applied to word blocks started at ordinary RF (mean score of ratings based on three baseline sessions: $\mathrm{RF}=5$ ); decreased to an average rating of 4.3 based on the sessions around 80 hours into treatment; increased to an average rating of 4.5 based on the sessions around 160 hours; and finally, after 240 hours, increased to a mean score of 6 (mean score of ratings based on three sessions after 240 hours: average to marked RF) (see the black bars in Figure 4). Considering all sessions, in 60 percent an RF score of 7 could be reached, and in 40 percent the RF score ranged between 3 and 7. Thus, Ms. A.'s ability to mentalize fluctuated within each session and in time came to reach levels as high as 7 .

For Ms. B., smaller changes took place. At baseline, overall RF ratings based on the session material were coded as low-level mentalization 


\section{Figure 5: Mean scores of ratings of the RF Scale applied to} sessions compared to the RF Scale applied to the AAl, changes in attachment representations and in SCL-90-R, Ms. B.

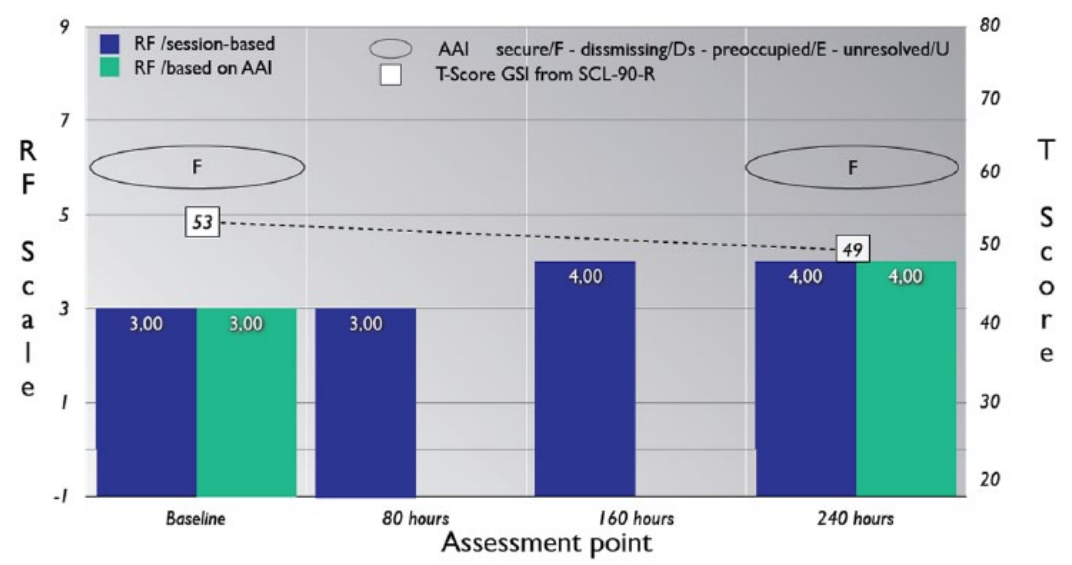

(mean score of ratings based on three baseline sessions: $\mathrm{RF}=3$ ); remained at a level of 3 around session 80; increased to a low to average level of RF (mean score of $\mathrm{RF}=4$ ) around session160; and remained at this level at 240 hours of treatment (see the black bars in Figure 5). All of Ms. B.'s session ratings ranged between 1 and 5 . When comparing the two cases, it becomes clear that a marked increase of RF took place between 160 and 240 hours and that Ms. A. showed more fluctuations throughout her sessions and reached overall higher levels of RF.

\section{External Validation: Comparison of Session-Based RF Ratings with RF Ratings Based on the AAI}

As the described method is a new application of the RF Scale, the session-based RF ratings were compared to RF ratings based on the "gold standard" AAI as a means of external validation, a method not hitherto implemented. The comparison was carried out as follows: In addition to ratings within each session in 150 -word blocks, an overall RF score was given to each session. The mean score of these overall ratings per assessment point is shown in Figures 4 and 5. They are compared to the RF ratings based on the AAI at baseline and after 240 hours. If available, follow-up interviews were conducted one year after treatment and rated for RF and AAI. At baseline, rather good correspondence was found for 
both patients between RF ratings based on the analytic sessions (mean scores) and independent RF ratings based on the AAI. At 240 hours, again the two rating procedures coincided well, but slightly different ratings for Ms. A. were given (see Figures 4 and 5). In summary, for Ms. A. the independent ratings corresponded well at baseline, an RF rating of 5 was given as the AAI-based score (i.e., a rating of $\mathrm{RF}=5$ in both methods), but the methods evaluated changes in RF differently: based on the therapy transcripts, an improvement in mentalizing capacities in the sessions was established, reflected by a mean RF score of 6 . Based on the AAI, a rating of 5 was given; hence, from this perspective, the average level of RF was maintained and did not change during psychotherapy. Follow-up data were available only for Ms. A., as Ms. B. could not be reached once her treatment had ended. Ms. A. further improved in RF based on the AAI at follow-up: at that point she received a score of 7 .

For Ms. B., the independent RF rater gave her a score of 3 at the beginning of treatment based on the AAI (i.e., ratings of RF $=3$ in both methods) and described an improvement to level 4 based on the AAI at 240 hours (i.e., ratings of RF $=4$ on both methods).

The different courses taken by the two cases will be discussed in greater detail. In Ms. A. a change in RF based on the AAI was found only in the long-term follow-up after treatment, while in Ms. B. changes in RF based on the AAI were found already at the end of treatment. However, in both cases changes in AAI-based RF seemed to follow session-based RF over the course of time.

If RF-AAI codings before and after 240 hours of psychoanalytic therapy are compared, Ms. B. showed higher single codings in the postinterview concerning difficult topics like rejection and loss. In the baseline interview the patient was unable to talk about rejection by her parents and in fact claimed that there was no rejection she could remember. Passive evasion was coded as no RF because the patient failed to elaborate on and mentalize about this difficult topic. In the post-interview, however, she remembered vividly several episodes of rejection by her mother. Furthermore, she was able to take her mother's perspective into account, even though she talked about painful memories and disappointment. Formerly unconscious memories were now accessible to reflection, and she was able to elaborate on her feelings and her psychological theory about her mother. This was the basis for the higher RF score. 
Ms. A. was already mentalizing at the average level before her psychoanalytic therapy. Her AAIs at baseline and after 240 hours are very similar, as in the course of both interviews RF broke down when she was asked to reflect on personal loss. After this breakdown she was unable to regain her reflective abilities and remained below average in both interviews. These results point to the hypothesis that issues around experiences of loss are too painful to be reflected on. Conflict-related breakdown of RF concerning loss has been described for depressed patients (Taubner et al. 2011).

At follow-up after treatment, Ms. A. maintained her ability to mentalize when reflecting on the impact of her grandmother's death and the emotions surrounding it. She was given a score of 6 on the RF Scale (above average to marked RF) because she was able to mentalize her ambivalence (shocked and sad vs. extremely angry) and reflect on the psychological impact of the experience on how she copes with death today (she works in a medical setting and has to deal with dying patients). Further, she could take different perspectives on her grandmother's loss by considering the reactions of her sister and her mother. All in all, the AAI-RF from the follow-up revealed marked changes in the quality of her psychological reasoning about attachment experiences, which led to a total score of 7 for this interview.

\section{Changes of Attachment Classifications on the AAI}

Ms. A.'s attachment classification at baseline was unresolved with respect to trauma, combined with an insecure preoccupied attachment representation (U/E). After 240 hours of psychotherapy, she was classified again as unresolved, with the secondary classification of dismissive (U/Ds). At follow-up she was classified secure with a tendency toward preoccupation (F4; see Figure 4).

Adults with the classification unresolved (U) show temporary lapses in the monitoring of reasoning or discourse during discussion of potentially traumatic events. Specifically, lapses in reasoning-for example, indications that a speaker believes that a deceased person is both dead and not dead - may indicate parallel but incompatible belief and memory systems regarding a traumatic event that have become dissociated. Lapses of monitoring of discourse, such as a sudden change into eulogistic speech, suggest the possibility of state shifts. By Main and Goldwyn's criteria (1994), interviewees should be classified as unresolved if during 
discussions of loss or abuse they show such lapses. Ms. A. showed in both interviews (pre- and post-) a failure to integrate the loss of her grandmother or her massively abusive experiences with a close relative. She felt that these experiences had influenced her personality in a maladaptive way. With respect to the abuse, she still struggled with emotions of disgust in romantic relationships, helplessness, and irrational guilt feelings. As for the loss of her grandmother, she still felt deserted by her, terribly missing her availability and love. Ms. A. stated herself that this experience of loss was not resolved at all. There are indications in both interviews of her disbelief that the grandmother is really dead.

In her secondary classification Ms. A. shifted from a preoccupied to a dismissive state of mind during therapy. Adults with the classification preoccupied (E) recount conflict-stricken events in an excessive, often nonobjective, and angry way and sometimes offer exaggerated pseudopsychological analyses or an oscillation between positive and negative evaluations, without being conscious of the inherent contradiction. In general, their language seems confused, unclear, and vague. In her interview before therapy, Ms. A. showed present anger toward her father. She felt emotionally neglected by him and reported pressure to achieve, as well as role-reversal with both parents. In summary, Ms. A. appeared enmeshed and gave the impression that she felt as if past experiences were occurring now and she was unable to distance herself from them. Interestingly, her anger changed during therapy; Ms. A. shifted to a more dismissive and idealizing state of mind with respect to both of her parents. Adults with the classification dismissive (Ds) give incoherent, incomplete accounts of their experiences and often show gaps in memory. As a defense against the surfacing of painful memories, they minimize the importance of attachment. They insist on normality and inner independence from others. Attachment figures are for the most part presented positively. Ms. A. insisted she had no memory of concrete episodes with her parents. Though she presented both in a positive way, she was unable to give concrete examples supporting this positive view of them. In contrast to her first interview, anger was no longer evident.

The AAI classification at follow-up changed to secure-autonomous with a tendency toward preoccupation (F4). The patient was able to present difficult experiences with primary caregivers in a more integrated manner. She neither idealized her parents nor accused them, as she had at the beginning of treatment. Especially when talking about her abusive 
experiences with a close relative, she now brought these memories up in an open manner without minimizing their effects. Now she was able to talk about the loss of her grandmother in an organized and resolved way. Although she was still very affected by the loss, there were no lapses of reasoning or of monitoring of the loss, indicating that she now fully realized that her grandmother was no longer alive. She was able to express her sadness.

At baseline and after 240 hours of psychotherapy, Ms. B. was classified with secure attachment representations (secure F). Adults with this classification give open, coherent, and consistent accounts of their childhood memories, regardless of whether they were positive or negative. They are able to integrate their experiences into a unitary whole and to reflect upon their accounts during the interview. They have free access to the topics asked about and show a feeling for balance. "Secure" discourse can be understood in terms of a capacity for fluidly shifting attention between memories and the maintenance of coherent discourse with the interviewer. At the beginning of treatment, Ms. B. showed a secure attachment representation with some dismissive features (F), meaning that she slightly idealized her mother. After 240 hours of psychotherapy, her secure inner working model of attachment remained, but now she presented an authentic picture that her childhood was difficult, including memories that her mother had hit her. Moreover, the painful loss of her grandmother was much more present than in the interview at the beginning of her treatment (see Figure 5 for the overall classifications).

Interestingly, Ms. B., whose RF increased throughout treatment by both measures, showed secure attachment at baseline, and maintained this attachment representation. Ms. A., whose attachment representation was unresolved at baseline, and remained disorganized after 240 hours of psychotherapy, was classified as secure at follow-up. Change in RF based on the AAI did not occur during treatment, but was seen only in the longterm follow-up. This improvement of AAI-based RF was preceded by a slight improvement of session-based RF during treatment.

\section{Changes in Symptom Severity on the SCL-90-R}

As an indicator of symptom severity, the General Severity Index from the SCL-90-R was compared from before treatment to the score after 240 hours of psychotherapy. Ms. A. described quite some symptom distress ( $t$ score of 67) at baseline, which even increased slightly over 
time ( $t$ score of 70). At follow-up, the symptom distress decreased a bit to a $t$ score of 66. In contrast, Ms. B. showed a rather low level of symptom severity both at baseline ( $t$ score of 53) and displayed a further reduction ( $t$ score of 49). The respective $t$ scores are shown in Figures 4 and 5.

Taking the changes in RF, attachment, and symptom severity together, it seems that Ms. B. achieved more changes during treatment than did Ms. A., who achieved changes only in the year after treatment.

\section{DISCUSSION}

Overall, the application of the RF Scale to psychoanalytic sessions appears to be a promising addition to the study of analytic process and mechanisms of change. The mentalizing abilities of patients within analytic sessions seem to adhere more to a "state" model than a "trait" one, as reflected by fluctuations within sessions and throughout the analytic process. However, using blinded ratings over several sessions of psychoanalytic psychotherapy, a continuous increase of patient's RF based on 150-word blocks was found over the course of 240 hours of psychoanalytic psychotherapy, and on the whole a good correspondence to a certified external rater's RF scoring based on the AAI was found. This result, though based only on two single-case studies, emphasizes the usefulness of the concept of RF as a potential mediator in psychoanalytic psychotherapies.

Our study has three main findings. The key finding is that RF can be applied to session material and leads to meaningful information on the analytic process. We have found quite fluctuating levels of RF within sessions and some interaction with the therapist's interventions. This was the case in every one of the twenty sessions we examined, particularly with regard to the therapist's interventions increasing the RF level during the session. Intuitively, the participating psychoanalysts gave transference interpretations at times when the RF performance of patients was low, a situation well documented in the session excerpts. At the same time, the finding that transference interpretations increase RF cannot be generalized; for example, mentalization-based treatment (Bateman and Fonagy 2004) focuses on other therapeutic interventions to increase RF. It remains an open question which interventions lead to an increase of RF. From the two cases examined here, however, it seems that various techniques, including transference interpretation, support mentalization: at first 
during sessions (with the therapist's help) and independently on the AAI. Future research needs to examine what types of intervention promote RF and what types lead to its decrease (questions, clarifications, interpretations, etc.). It also needs to be determined whether the patient's higher RF level following a therapist's intervention can be understood as an imitation of the therapist's RF or rather as the result of a dynamic process in which material that earlier could not be mentalized becomes more easily elaborated mentally following the intervention.

Our second finding is that session-based RF changes may precede AAI-based RF changes. This was seen in both of the treatments studied. We interpret this interesting finding by understanding AAI-based assessment of RF as a more conservative approach that captures only strong and significant changes in a "trait"-like RF, while seeing session-based assessment as more sensitive to small fluctuations and volatile "state"-like manifestations of RF. Particularly in Ms. A., differences between session-based ratings and AAI-ratings could be due to an avoidance of certain topics during therapy or to a sampling issue: because her ten sessions were randomly selected for coding, any that may have dealt with loss-related issues could have been excluded by chance. Nonetheless, this result points to the main difference between session-based and AAI-based RF codings. The AAI consists of a standardized set of questions, where no supporting interventions are given (e.g., questions that might encourage $\mathrm{RF}$ ), that would allow the tracking of changes in RF concerning specific attachment-related topics. Meanwhile, RF may improve during the course of treatment in areas not covered by the AAI. Another hypothesis might be that the AAI fails to capture all relevant changes in RF because the protocol focuses strongly on past relationships. So while Ms. A. was showing general improvement in RF during the therapeutic sessions, over a rather long period, she continued to have difficulty reflecting on losses of significant attachment figures on the AAI (a difficulty that may not hinder the therapeutic process in other regards).

Our third finding is that changes in RF seem to depend on a complex interplay of (a) baseline RF level, (b) attachment security, (c) general level of personality functioning, and (d) therapeutic process. The two cases presented had a relatively high baseline level of RF, which was reflected by the indication for psychoanalytic psychotherapy; hence, large changes in structural measures like RF were not expected. In contrast, in patients with borderline personality disorder, who have a lower level of 
personality functioning, larger changes in RF can be expected and have been found. Regarding level of personality functioning, both of our patients were diagnosed at good-to-moderate level of structural integration, so this alone cannot explain their different courses. Ms. B. showed an increase in session-based RF earlier in treatment (after 160 hours). No changes in session-based RF were found with Ms. A. during the first 160 hours; her increase in RF took place after 240 hours. In terms of attachment classification, it is very likely that Ms. B., starting treatment with secure attachment, had different prerequisites for change than Ms. A. The latter was classified with disorganized attachment representations and her trauma remained unresolved for a long time, until the end of treatment. It can be hypothesized that disorganized attachment might impede more drastic changes in RF. This association appears consistent with Bowlby's original predictions (1980) regarding psychiatric instability as a potential response to the death of attachment figures. He postulated that chronic mourning was pathological because the individual remains in a state of mind that was unable (consciously and unconsciously) to reorganize, mentalize, and reintegrate his or her mental representation of attachment to the deceased. Mourning begins, but it is not completed. Yet the individual's hope for a reunion, for comfort and care from the attachment figure, cannot be realized and may be accompanied by psychiatric disorders such as anxiety, depression, or personality disorders (Buchheim and George 2011). This is exactly what we found in the case of Ms. A. Only during follow-up did she show a new ability to mentalize the loss of her grandmother, thus yielding a higher RF rating in general. In parallel, the attachment classification changed to "earned secure attachment," which may be interpreted as meaning that AAI-based RF and attachment classification both change at a slower rate than session-based RF. Lastly, various aspects may contribute to the different treatment processes of the two patients. Ms. B. showed little symptom severity at baseline or over time and demonstrated a critical change in RF in the sessions. With Ms. A. it appears that at the beginning of treatment she experienced a negative therapeutic process, which is mirrored by the decrease in session-based $\mathrm{RF}$ and an increase in symptom burden (measured by the GSI). At 240 hours, with the help of certain interventions, she increased her RF temporarily during the sessions, but she could not maintain this increase on the AAI. The therapist's interventions might have supported her ability to mentalize within the sessions, but she could not use this mentalization 
independently from her therapist when examined on the AAI. This might be explained by the very abstinent procedure of the AAI protocol, which is quite different from the dialogue in a therapeutic session. Finally, at follow-up the severity of Ms. A.'s symptoms had decreased, AAI-based RF was increased and appeared consolidated, and attachment security was attained.

In summary, our main conclusion is that session-based RF can be a useful process measure and should be tested as a mediator of symptomatic and AAI-RF changes, whereas AAI-based RF is useful as an outcome measure. However, a combination of of the two measures seems rewarding for process-outcome study designs. The perspectives from within the treatment and externally from the AAI complement each other.

Future studies need to replicate these findings with larger samples, as this study of two cases can be seen as merely exploratory. Also, our procedure could be investigated in multi-method approaches, for example, combining it with results from other process measures or instruments examining changes in personality functioning.

\section{REFERENCES}

AlLeN, J.G. (2006). Mentalizing in practice. In Handbook of MentalizationBased Treatment, ed. J.G. Allen \& P. Fonagy. Hoboken, NJ: Wiley, pp. 3-30.

Bateman, A.W., \& Fonagy, P. (2004). Mentalization-based treatment of BPD. Journal of Personality Disorders 18:36-51.

Bernbach, E.J., Muran, J.C., Slade, A., \& Tuber, S. (2000). Reflectivefunctioning and the therapeutic relationship: Understanding change in a brief relational psychotherapy. Paper presented to the Society for Psychotherapy Research, Chicago, June.

BowlbY, J. (1980). Attachment and Loss: Vol. 3. Loss. New York: Basic Books.

BuchHeim, A., \& George, C. (20I I). Attachment disorganization in borderline personality disorder and anxiety disorder. In Disorganized Attachment and Caregving, ed. J. Solomon \& C. George. New York: Guilford Press, pp. 343-382.

CHOI-KAIN, L.W., \& GUNDERSON, J.G. (2008). Mentalization: Ontogeny, assessment, and application in the treatment of borderline personality disorder. American Journal of Psychiatry 165:1127-1135.

Fischer-Kern, M., Doering, S., Taubner, S., Hörz, S., Zimmermann, J., Rentrop, M., Schuster, P., Buchreim, P., \& Buchreim, A. (2015). Transference- 
focused psychotherapy for borderline personality disorder: Change in reflective function. British Journal of Psychiatry 206:1-2.

FonaGy, P., \& BATEMAN, A.W. (2006). Mechanisms of change in mentalizationbased treatment of BPD. Journal of Clinical Psychology 62:411-430.

Fonagy, P., Gergely, G., JuRISt, E., \& TARGet, M. (2002). Affect Regulation, Mentalization, and the Development of the Self. New York: Other Press.

Fonagy, P., Moran, G.S., Edgcumbe, R., Kennedy, H., \& Target, M. (1993). The roles of mental representations and mental processes in therapeutic action. Psychoanalytic Study of the Child 48:9-48.

Fonagy, P., Target, M., Steele, H., \& Steele, M. (1998). Reflective Functioning Scale. Unpublished manual, University College London.

Franke, G.H. (1995). SCL-90-R. Die Symptom-Checkliste von Derogatis: Deutsche 48. Version. Göttingen: Beltz Test.

George, C., Kaplan, N., \& Main, M. (1985). The Berkeley Adult Attachment Interview. Unpublished protocol, University of California, Berkeley.

GrenYeR, B.F., \& MidDleby-ClementS, J. (2003). Growth in reflective functioning and mastery over the course of psychotherapy. Paper presented at the Clinical Attachment Research 26th International Workshop on Empirical Research in Psychoanalysis, Ulm, Germany.

Gullestad, F.S., Johansen, M.S., Høglend, P., Karterud, S., \& Wilberg, T. (2013). Mentalization as a moderator of treatment effects: Findings from a randomized clinical trial for personality disorders. Psychotherapy Research: Journal of the Society for Psychotherapy Research 23:674689.

GulLESTAD, F.S., \& WILBERG, T. (20I I). Change in reflective functioning during psychotherapy: A single-case study. Psychotherapy Research: Journal of the Society for Psychotherapy Research 21:97-111.

Hesse, E. (2010). The Adult Attachment Interview: Protocol, method of analysis, and empirical studies. In Handbook of Attachment: Theory, Research, and Clinical Applications, ed. J. Cassidy \& P. Shaver. 2nd ed. New York: Guilford Press, pp. 552-598.

ISPHORDING, S. (2009). Psychoanalytische Therapieforschung: Die Reflexive Funktion im Verlauf psychoanalytischer Langzeittherapie. Unpublished diploma thesis, Ludwig-Maximilian-Universität, Munich.

Johansson, P., Høglend, P., Ulberg, R., Amlo, S., Marble, A., Bogwald, K.-P., Sørbye, O., SJAAstad, M.C., \& Heyerdahl, O. (20l0). The mediating role of insight for long-term improvements in psychodynamic therapy. Journal of Consulting \& Clinical Psychology 78:438-448.

Jones, E.E. (1985). Manual for the Psychotherapy Process Q-sort. University of California, Berkeley. 
Josephs, L., Anderson, E., Bernard, A., Fatzer, K., \& Streich, J. (2004). Assessing progress in analysis interminable. Journal of the American Psychoanalytic Association 52:1185-1214.

KARLSSON, R. (2005). Reflective-functioning during the process and in relation to outcome in cognitive-behavioral therapy, interpersonal psychotherapy and brief psychodynamic psychotherapy. Umeå Psychology Supplement Reports 10:1-31.

KarlsSON, R., \& KeRMOTT, A. (2006). Reflective-functioning during the process in brief psychotherapies. Psychotherapy 43:65-84.

Katznelson, H. (2014). Reflective functioning: A review. Clinical Psychology Review 34:107-117.

Levy, K.N., Meehan, K.B., Kelly, K.M., Reynoso, J.S., Weber, M., Clarkin, J.F., \& KERNBERG, O.F. (2006). Change in attachment patterns and reflective function in a randomized control trial of transference-focused psychotherapy for borderline personality disorder. Journal of Consulting \& Clinical Psychology 74:1027-1040.

MaIN, M., \& Goldwrn, R. (1994). Adult Attachment scoring and classification system. Unpublished classification manual, University of California, Berkeley.

Müller, C., Kaufhold, J., Overbeck, G., \& Grabhorn, R. (2006). The importance of reflective functioning to the diagnosis of psychic structure. Psychology \& Psychotherapy 79:485-494.

Müller-GöttKen, T., White, L.O., Klitzing, K., \& von Klein, A.M. (20l4). Reflexive Kompetenz der Mütter als Prädiktor des Therapieerfolgs mit psychoanalytischer Kurzzeittherapie im Alter von 4-10 Jahren. Praxis der Kinderpsychologie \& Kinderpsychiatrie 63:795-811.

OPD TASK ForCE (2008). Operationalized Psychodynamic Diagnostics OPD2: Manual of Diagnosis and Treatment Planning. Cambridge, MA: Hogrefe.

Rudden, M., Milrod, B., Target, M., Ackerman, S., \& Graf, E. (2006). Reflective functioning in panic disorder patients: A pilot study. Journal of the American Psychoanalytic Association 54:1339-1343.

Segal, Z.V., Williams, J.M.G., \& Teasdale, J.D. (2002). Mindfulness-Based Cognitive Therapy for Depression: A New Approach to Preventing Relapse. New York: Guilford Press.

ShapiRo, S.L., Astin, J.A., BISHOP, S.R., \& Cordova, M. (2005). Mindfulnessbased stress reduction for health care professionals: Results from a randomized trial. International Journal of Stress Management 12:164-176.

Stegmaier, S. (2007). Die Entwickung der Mentalisierungsfähigkeit im Verlauf psychoanalytischer Therapien am Beispiel zweier Patientinnen: 
Eine empirische Untersuchung. Unpublished diploma thesis, LudwigMaximilians-Universität, Munich.

SzECsÖDY, I. (2008). A single-case study on the process and outcome of psychoanalysis. Scandinavian Psychoanalytic Review 31:105-113.

Taubner, S., Hörz, S., Fischer-Kern, M., Doering, S., Buchheim, A., \& ZimmermanN, J. (2013). Internal structure of the Reflective Functioning Scale. Psychological Assessment 25:127-135.

Taubner, S., Kessler, H., Buchreim, A., Kächele, H., \& Staun, L. (20II). The role of mentalization in the psychoanalytic treatment of chronic depression. Psychiatry, 74(1), 49-57.

Taubner, S., Schulze, C., Kessler, H., Buchheim, A., Kächele, H., \& Staun, L. (20I5). Veränderungen der mentalisierten Affektivität nach 24 Monaten analytischer Psychotherapie bei Patienten mit chronischer Depression. Psychotherapieforum.

Vermote, R., Lowyck, B., Luyten, P., Vertommen, H., Corveleyn, J., Verhaest, Y., Stroobants, R., Vandeneede, B., Vansteelandt, K., \& Peuskens, J. (2010). Process and outcome in psychodynamic hospitalization-based treatment for patients with a personality disorder. Journal of Nervous \& Mental Disease 198:110-115.

Susanne Hörz-Sagstetter

Psychologische Hochschule Berlin

Am Köllnischen Park 2

10179 Berlin

GERMANY

E-mail: s.hoerz@psychologische-hochschule.de 